\title{
Comparative Analysis of Depth of Meditation Between Long-Term and Short Term Meditators using Electroencephalography
}

\author{
Dipti Magan, Raj Kumar Yadav*, Kishore Kumar Deepak
}

\section{Dipti Magan, Raj Kumar Yadav*, Kishore Kumar Deepak}

Department of Physiology, All India Institute of Medical Sciences, New Delhi-110029, INDIA.

\section{Correspondence}

\section{Dr. Raj Kumar Yadav, MD}

Professor, Department of Physiology, All India Institute of Medical Sciences, New Delhi-110029, INDIA.

Phone: +01126546461

Email: raj3kr@gmail.com

History

- Submission Date: 14-07-2018

- Review completed: 20-09-2018

- Accepted Date: 10-10-2018.

DOI : 10.5530/ijcep.2018.5.4.18

\section{Copyright}

(C) 2018 Phcog.Net. This is an openaccess article distributed under the terms of the Creative Commons Attribution 4.0 International license.

\begin{abstract}
Background and Aim: The present study was designed to evaluate the brain electrical activity among Long-term meditators (LTMs) and Short-term meditators (STMs) using Electroencephalography (EEG). Methods: This prospective study involved 40 mins of EEG recordings, carried out twice i.e. at Day 0 (Baseline) and at Day 10 (During meditation) in thirty-four (34) participants. Twelve (12) apparently healthy male, right-handed, practicing preksha meditation (Since $>5$ years, at least 5 days a week) were recruited as long-term meditators; Twelve (12) subjects who attended the 10-days short-term yoga-based lifestyle intervention program were recruited as short-term meditators; Ten (10) male healthy controls, who did not participate in any dietary/yoga intervention were recruited among the staff members. The EEG waves were analyzed at Day 0 and at Day 10 using Wilcoxon Signed Rank test and Repeated measured ANOVA respectively. One-way ANOVA was used for the baseline EEG waves activity comparison among LTMs vs. STMs vs. Controls. The response to photic stimulation was also assessed during meditation. Results: Significant different patterns of alpha and theta waves $(p<0.05)$ activity was observed for LTM vs. STM during meditation vs. baseline. Also, significant different baseline EEG waves $(p<0.05)$ were observed among LTM vs. STM vs. Controls. Conclusion: Findings may be useful in defining the basic EEG features to differentiate meditators based on the duration of practice of meditation and depth of meditation.

Key words: Meditation, Electroencephalography, Long-term meditators, Short-term meditators, Alpha waves, Theta waves.
\end{abstract}

\section{INTRODUCTION}

Nowadays, yoga-meditation is recommended as a complimentary therapy for stress and chronic diseases management that relaxes mind. ${ }^{[1]}$ It is of great significance to investigate the mental traits of an individual during the meditation state. Electroencephalography (EEG) is one of the primary methods to evaluate the various physiological activities such as state of wakefulness, sleep and various attentive tasks that include meditation. Studies have been conducted during different types of meditation practices and showed varying patterns of enhanced alpha activity ${ }^{[2-4]}$ and theta activity ${ }^{[2,3]}$ as well as gamma activity. ${ }^{[5,6]}$ These differences might be due to the different duration of meditation practice, type and depth of meditation. There is a paucity of literature to quantify the depths of meditative states. Numerous studies have indicated an overall slowing down of brain rhythms after meditation. ${ }^{[2-4,7-12]}$

Therefore, the present study attempts to explore the effects of long-term and short-term meditation practice on different EEG waves at rest and during meditation. Also, to quantify the meditative states, baseline EEG waves were evaluated among long-term meditators $v s$. short-term meditators vs. controls.
The photic stimulation was also assessed to affirm meditation that document sensory attenuation.

\section{MATERIALS AND METHODS}

This was a prospective interventional study with pre- post design. A total of Fifty-four meditators and healthy controls were initially enrolled in the study between July 2012 and December 2013. Out of the 54 participants, Twelve declined to participate and Eight did not come for tests for the last day of the intervention, i.e. at day 10 , therefore, a total of 34 participants completed the study. All long-term meditators were male, right handed and were recruited from 'Adhyatma Sadhna Kendra', Chattarpur Ashram, New Delhi. They were practicing meditation since 11 years (Median; range 9 to 50 years), for 45.0 mins (Median; range 30 to 60 mins) per day and at least 5 days (Median; range 5 to 7 days) a week. Subjects who were willing and had ability to attend the 10-days (Short-term) lifestyle intervention program at Integral Health Clinic (IHC), Department of Physiology, All India Institute of Medical Sciences, New Delhi, India, were recruited as short-term meditators $(\mathrm{n}=12 ; 6$ males and 6 females. The control group included Ten healthy male subjects who
Cite this article: Magan D, Yadav RK, Deepak KK. Comparative Analysis of Depth of Meditation Between Long-Term and Short Term Meditators using Electroencephalography. Int J Clin Exp Physiol. 2018;5(4):178-83. 
had not attended any such program in the past 1 year and were recruited among the hospital staff and patients' attendants. Controls were enrolled for the baseline comparison of EEG waves activity with LTMs and STMs. Exclusion criteria included physically challenged subjects who were unable to perform the yogic exercises, major neurological or medical illness, history of serious heart disease (Heart attack, angina, cardiac surgery or congestive heart failure), serious mental illnesses and pregnant woman.

The study was conducted in accordance with the Declaration of Helsinki and was approved by the appropriate local ethics committee and Institutional Review Board. All participants provided their informed consent. The trial was registered at Clinical Trial Registry India (CTRI/2009/091/000727).

The pretested. ${ }^{[13]}$ IHC intervention consists of a short-term lifestyle intervention program that includes yogasana (Physical postures), breathing exercises, meditation, group discussions and individual advice on stress management, diet and physical activity in addition to group support. Each session lasted about $2 \mathrm{~h}$ per day for 10 days spread over 2 weeks. Routine clinical and laboratory variables were measured before and after the intervention.

Changes in the brain electrical activity was assessed using Nineteen channel digital EEG recording system (NEUROWERK, Sigma Medizin-Technik GmbH, Germany) with $\mathrm{Au}-\mathrm{AuCl}$ (Gold) electrodes using International 10-20 montage system, reference to the linked ear lobes. EEG recordings were carried out twice i.e. at baseline (Day 0) and during meditation (Day 10). During baseline (Day 0), recording was done for approximately 40 mins with eyes opened and eyes closed. At day 10, during meditation, EEG recording was carried out for approximately 40 mins with eyes closed. The Forty minutes (40 mins) of EEG recording during meditation was divided into four phases of time window i.e. Phase $1\left(\mathrm{P}_{1}\right)=$ first 10 mins of meditation; Phase $2\left(\mathrm{P}_{2}\right)=$ next $10 \mathrm{mins}$ (20 mins) of meditation; Phase $3\left(\mathrm{P}_{3}\right)$ = further next 10 mins (30 mins) of meditation; Phase $4\left(\mathrm{P}_{4}\right)=$ last 10 mins (40 mins) of meditation to observe the inter-phase comparison of different EEG waves dominance on the basis of absolute power. The EEG signals were quantified using frequency spectral analysis for each condition. Artifact-free epochs were selected for every $5 \mathrm{~min}$ of EEG recording for each condition and then, a mean FFT calculation was done to produce a frequency spectrum. The different band frequencies were identified as alpha $(8.0-12.9 \mathrm{~Hz})$, theta (4.0-7.9 Hz), beta $(13.0-29.9 \mathrm{~Hz})$ and delta $(<4 \mathrm{~Hz})$. The quantitative report shows absolute power $\left(\mu \mathrm{V}^{2}\right)$ of different EEG waves. The EEG recording for control group was carried out at Day 0 (baseline). The study design flowchart is shown in Figure 1. All EEG recordings were carried out between 8:30 and 9:30 AM.

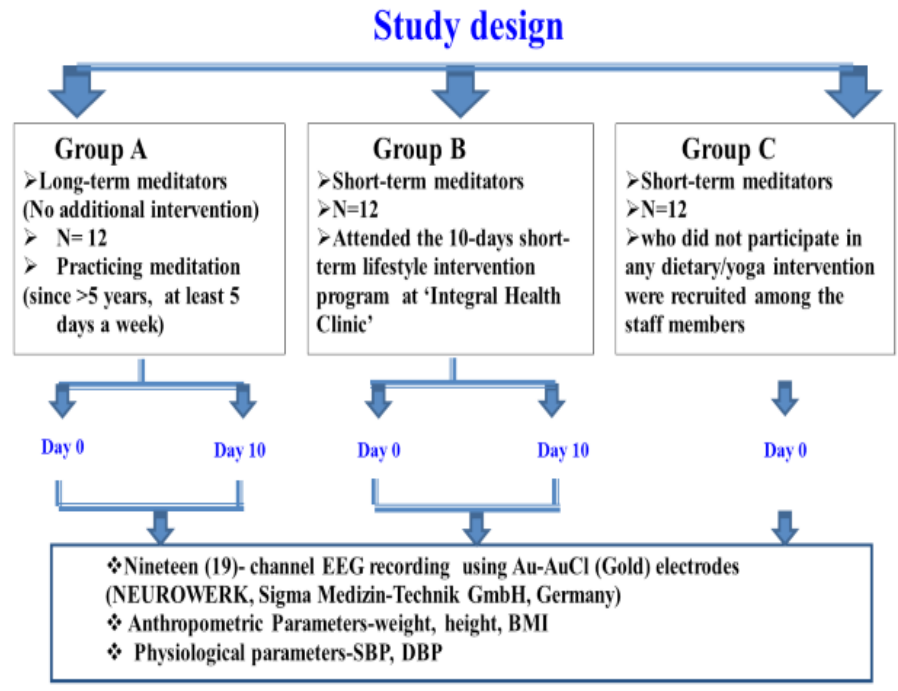

Figure 1: Study Design Flow Chart.

\section{Statistical analysis of data}

SPSS version 20.0 (SPSS Software Inc., Chicago, IL, USA) was used for statistical analysis. Using pre-post design, data was analyzed to compare the different EEG waves activity at Day 0 (Baseline; eyes closed $v s$. eyes open) using 'Wilcoxon Signed Rank' test. 'Repeated measures ANOVA' with Bonferroni correction was applied at Day 10 i.e. during different phases of meditation $v s$. initial resting eyes closed condition. One-way ANOVA was applied for the baseline comparison for different EEG waves among long-term meditators $v s$. short-term meditators $v s$. controls. All the data were expressed as median (Range).

\section{RESULTS}

Demographic characteristics for long-term meditators, short-term meditators and controls are shown in Table 1. At Day 0 (At rest; eyes closed $v s$. eyes opened), alpha absolute power was significantly higher for long-term meditators $(p<0.001)$, short-term meditators $(p=0.011)$ and for controls $(p<0.001)$. In addition, theta absolute power was significantly higher $(p<0.001)$ for long-term meditators at Day 0 (Table 2). Beta power was significantly lower $(p<0.001)$ both in long-term meditators and for controls $(p=0.030)$ and no significant difference was observed for delta wave activity at Day 0 (Table 2).

Table 1: Demographic characteristics of long-term meditators, short-term meditators and controls

\begin{tabular}{|c|c|c|c|c|c|c|c|}
\hline Variable & Long-term meditators & Short-term meditators & Controls & $\begin{array}{l}\text { Overall } \\
\text { p-value }\end{array}$ & $\begin{array}{c}\mathrm{P}_{1} \\
\text { LTM vs. STM }\end{array}$ & $\begin{array}{c}\mathrm{P}_{2} \\
\text { STM vs. } \\
\text { CONT }\end{array}$ & $\begin{array}{c}\mathrm{P}_{3} \\
\text { LTM vs. } \\
\text { CONT }\end{array}$ \\
\hline $\mathrm{N}$ & 12 (males) & 12 (6 females; 6 males) & 10 (males) & & & & \\
\hline Age (years) & $35.0(30.0-74.0)$ & $28.5(2-1.0-02.0)$ & $35.0(27.0-55.0)$ & & & & \\
\hline BMI (Kg/m2) & $21.62(20.36-28.84)$ & $26.79(21.4-28.61)$ & $25.77(22.32-28.22)$ & $p<0.001$ & $p<0.001$ & 0.05 & $p<0.001$ \\
\hline \multicolumn{8}{|l|}{$\begin{array}{l}\text { Blood Pressure } \\
\quad(\mathrm{mmHg})\end{array}$} \\
\hline Systolic & $116.0(110.0-124.0)$ & $122.0(112.0-146.0)$ & $118.0(106.0-128.0)$ & 0.02 & 0.008 & 0.01 & 0.053 \\
\hline Diastolic & $78.0(64.0-86.0)$ & $80.0(60.0-96.0)$ & $78.0(72.0-84.0)$ & 0.07 & 0.051 & 0.05 & 0.73 \\
\hline
\end{tabular}

All the values are in median (range). One way ANOVA is used for the comparison among Long-term meditators vs. Short-term meditators vs. Controls. $\mathrm{P}_{1}=$ Long-term meditators vs. Short-term meditators; $\mathrm{P}_{2}=$ Short-term meditators vs. Controls; $\mathrm{P}_{3}=$ Long-term meditators vs. Controls 

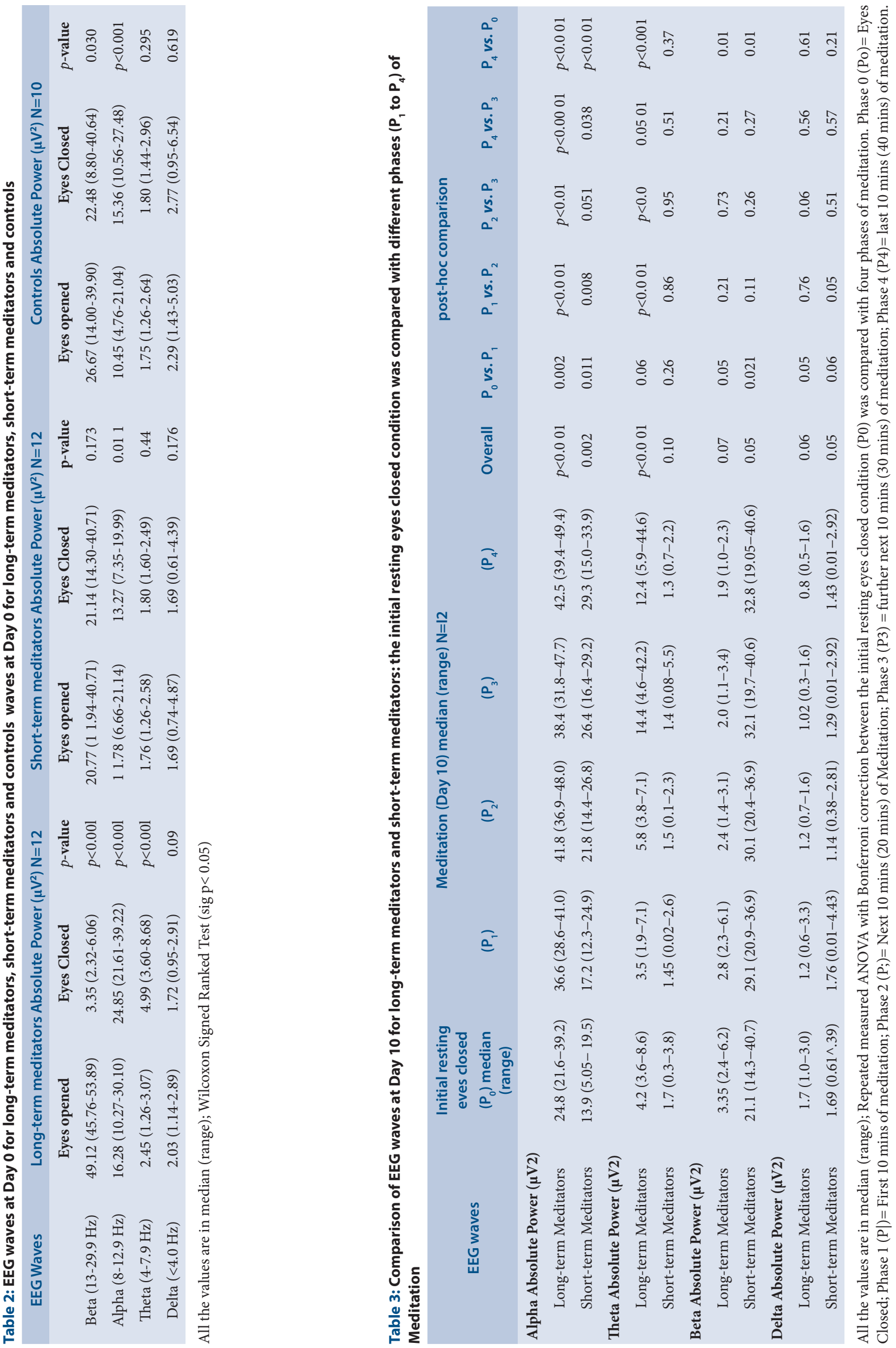
Analysis of EEG waves activity at Day 10 for LTM vs. STM i.e. different phases $\left(\mathrm{P}_{1}-\mathrm{P}_{4}\right)$ of meditation was compared with the initial resting eyes closed condition (Table 3$)$. Alpha $(p<0.001)$ and theta $(p<0.001)$ absolute power were significantly higher throughout the different different phases of meditation for LTM vs. STM (Table 3; Figure 2). On closer examination, long-term meditators showed significantly $(p=0.04)$ higher alpha waves activity in the posterior regions $v s$. frontal regions whereas theta activity was maximum in temporo-central regions with significant $(p=0.05)$ right central theta activity during meditation (Figure 2 ). On the other hand, short-term meditators showed significantly higher beta $(p<0.001)$ and alpha $(p<0.001)$ absolute powers during different stages of meditation $v s$. initial resting eyes closed condition (Table 3; Figure 1). The representative EEG recording and frequency spectrum for a long-
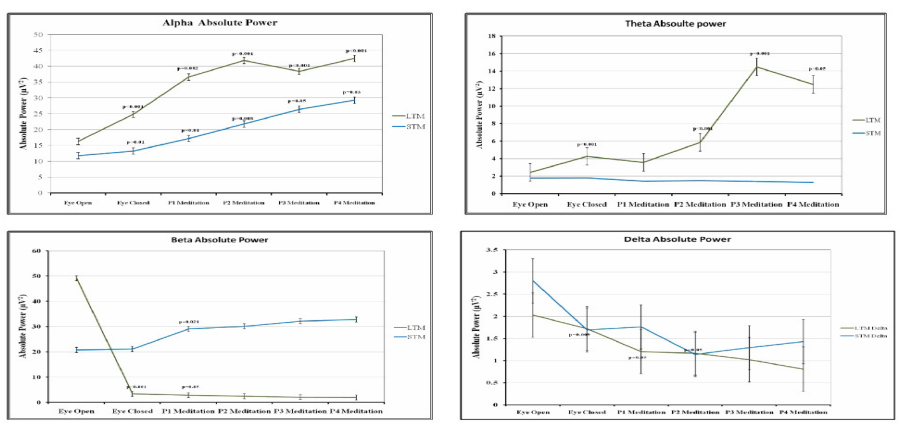

Figure 2: Graphical representation of different EEG waves for long-term meditators vs. Short-term meditator.
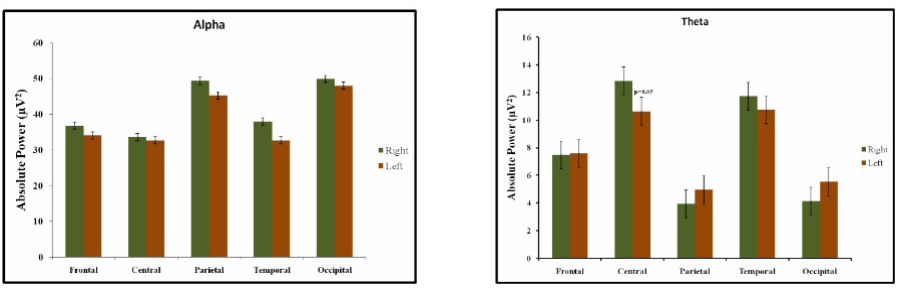

Figure 3: Graphical representation of alpha and theta EEG wave distribution across different regions during meditation for long-term meditators.
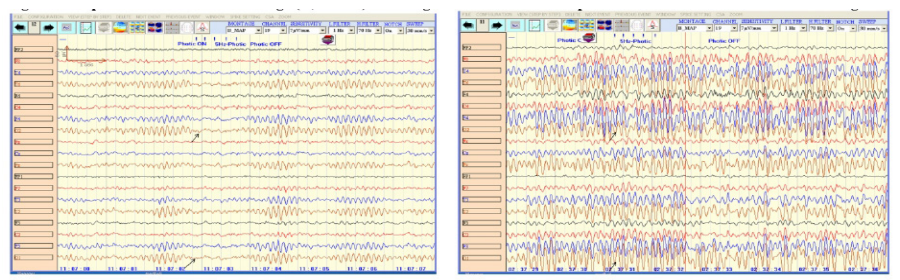

(A) At baseline with photic stimulation (arrows marked)
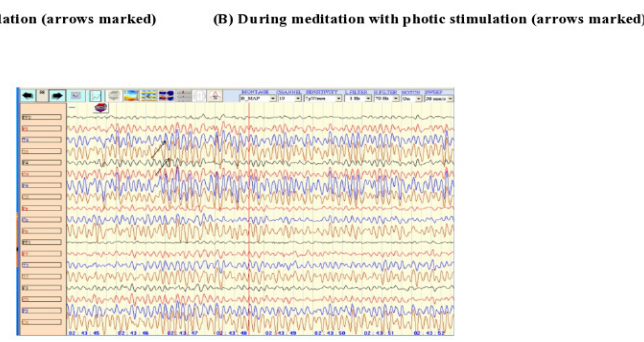

(C) During Meditation (Theta Waves) (Arrows showing Theta waves at T4, T6 Electrodes)

Figure 4: Representative EEG recording (A, B \& C) of a long-term meditator with photic stimulation at baseline and during meditation.

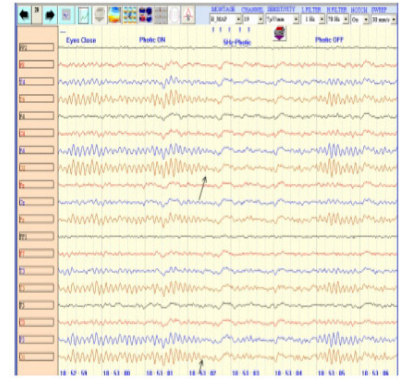

(A) At baseline (Day 0) with photic stimulation (arrows marked)

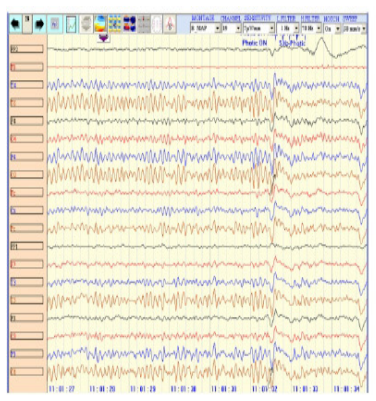

(B) During meditation with photic stimulation (arrows marked)
Figure 5: Representative EEG recordings (A \& B) of a short-term meditator with photic stimulation at baseline and during meditation.

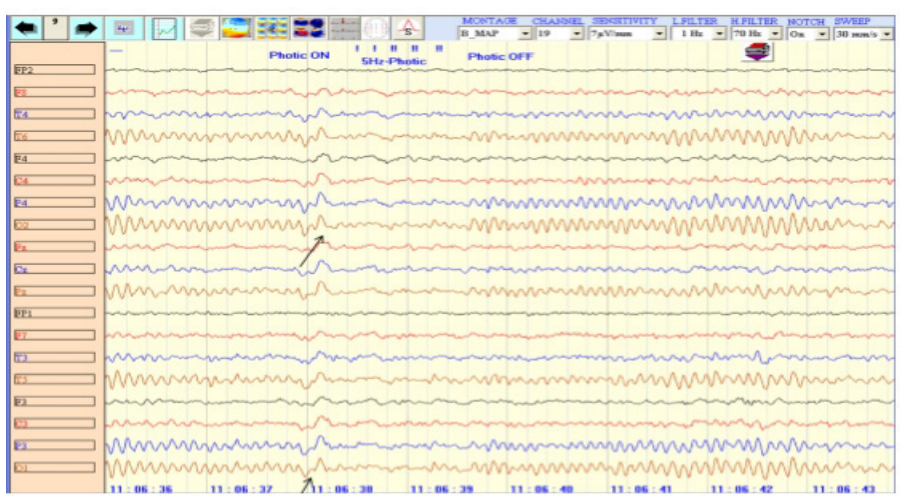

Figure 6: Representative EEG recording of a control with photic stimulation (arrows marked) at baseline.

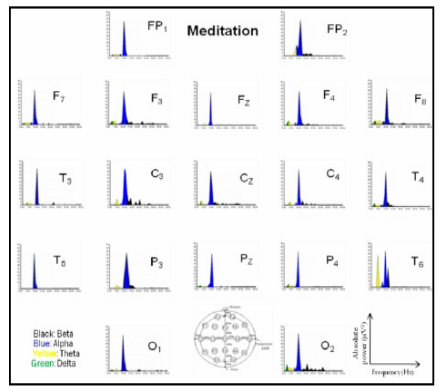

(A) Initial stages of Meditation

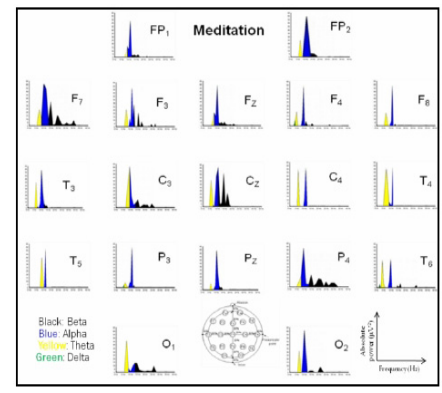

(B) Later stages of Meditation
Figure 7: Frequency Spectrum for EEG waves of a long-term meditator

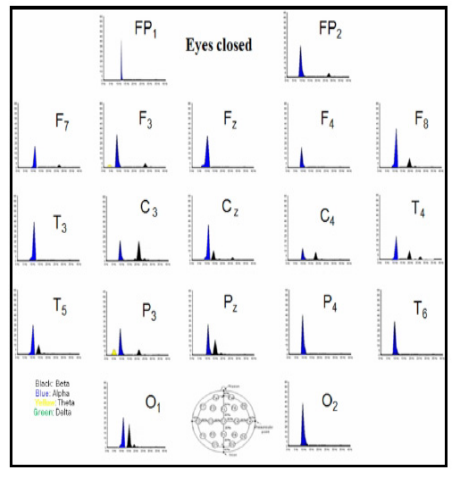

(A) Eyes closed

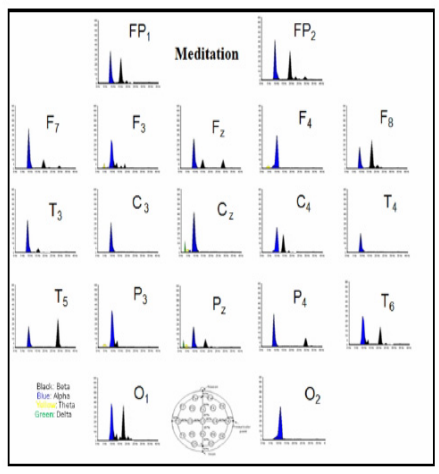

(B) During meditation
Figure 8: Frequency Spectrum for EEG waves of a Short-term meditator 
term meditator, short-term meditator and for controls are shown in Figure 4 (a, b and c), Figure 5 ( $a$ and b), Figure 6, Figure 7 ( $a$ and b) and Figure 8 ( $a$ and $b$ ) respectively.

Notably, comparison of analysis of depth of meditation was evaluated on the basis of baseline (Eyes closed) EEG waves activity and on alphablockage during meditation among long-term meditators $v$ s. short-term meditators $v s$. controls. Significant baseline differences were observed for different EEG waves activity among long-term meditators $v$ s. short-term meditators $v s$. controls (Table 4$)$. At baseline, alpha $(p<0.001)$ and theta $(p=0.022)$ absolute powers were found to be significantly higher whereas beta $(p<0.001)$ and delta $(p=0.026)$ absolute powers were significantly lower in long-term meditators when compared to short-term meditators and controls. Short-term meditators showed significantly lower delta absolute power when compared to controls (Table 4). Also, EEG recordings showed no alpha blockage for long-term meditators when compared to short-term meditators Figure 4 (a, b and c); Figure 5 ( $a$ and b).

\section{DISCUSSION}

Present study has highlighted the analysis of depth of meditation on the dominance of EEG waves activity during different phases of meditation (As described briefly in methodology section). Study findings have shown significant increase in alpha and theta waves activity during different phases of meditation in long-term meditators vs. short-term meditators $v s$. controls. During the initial phases of meditation (Phase 1 and phase 2), both alpha and theta waves activity was significantly and consistently higher from initial resting eyes closed condition that probably reflects relaxation, awareness, conscious state and affective processing. ${ }^{[1-4,8-11]}$ More importantly, during the middle phases of meditation i.e. from phase 2 to phase 3 , alpha waves were significantly replaced by theta waves, correlating positively and proportionately with the depth of the meditation experience for long term meditators. Further, in last phases of meditation i.e. from phase 3 to phase 4 , again alpha waves were consistently higher and there was 'switching off' irrelevant network for the maintenance of focused internalized attention and inhibition of inappropriate information ${ }^{[14]}$ and this was affirmed in our study by giving photic stimulation as shown in Figure 4(a,b and c); Figure 5(a and b). On further analysis during meditation, long-term meditators showed higher theta wave activity in temporal-central areas with significant right central brain region probably reflecting increased cognitive processing and awareness whereas alpha wave activity was higher in the posterior regions $v s$. frontal regions characterized by large rhythmic waves, which may be associated with awareness with relaxation as shown in Figure 3.

On the other hand, short-term meditators showed higher beta activity along with alpha activity during meditation. The increase in alpha activity likely relates to relaxation. ${ }^{[1-4,14,15]}$ Present study also showed increased beta activity along with increased alpha activity for short-term meditators which likely relates to increased alertness, awareness with relaxation. As expected, in present study, meditation produced little change in delta activity (Sleep or pathological processes). The above results concur with previous studies reporting EEG patterns for alpha $a^{[1-4,14,15]}$ or beta ${ }^{[16,17]}$ but detailed comparative analysis of EEG waves during different phases of meditation for LTMs $v s$. STMs are studied most probably or the first time in the present study.

EEG biorhythms are fundamentally different for long-term meditators, which predisposed the beneficial effects of long-term meditation practices. The remarkable differences in baseline EEG activity in long-term meditators provide the good impact to deliver the substantial evidences to confirm their long-term and regular practice of meditation which is different from short-term meditators and controls.

Short-term meditators also showed upgrading in their EEG activity as early as 10 days, observed by increase in alpha wave activity during meditation, which indicates relaxation and awareness. The presence of alpha block and unchanged theta activity are probably due to short term meditation exposure. The alpha block and significantly higher theta activity may be considered as a measure of depth, duration of involvement in active meditation experiences. It is apparent from the findings that long-term meditation practice has a positive impact in modulating brain activity such that it helps the individuals to overcome day to day stress.

A major limitation of the current study was an inability to do further EEG analysis with highly advanced digital EEG system with higher number of channels and only male subjects were recruited in long-term meditation group. Despite this, the results remain highly valuable due to following strengths. First, this study remains the first study in Indian population that evaluated the EEG activity among long-term meditators $v s$. short-term meditators vs. controls. Secondly, it is important to know if subjects were actually practicing meditation (Quantification of meditative states), which we tried to validate by continuous EEG recording with photic stimulation during meditation in this study.

Table 4: Baseline EEG waves in Long-term meditators vs. Short-term meditators vs. Controls

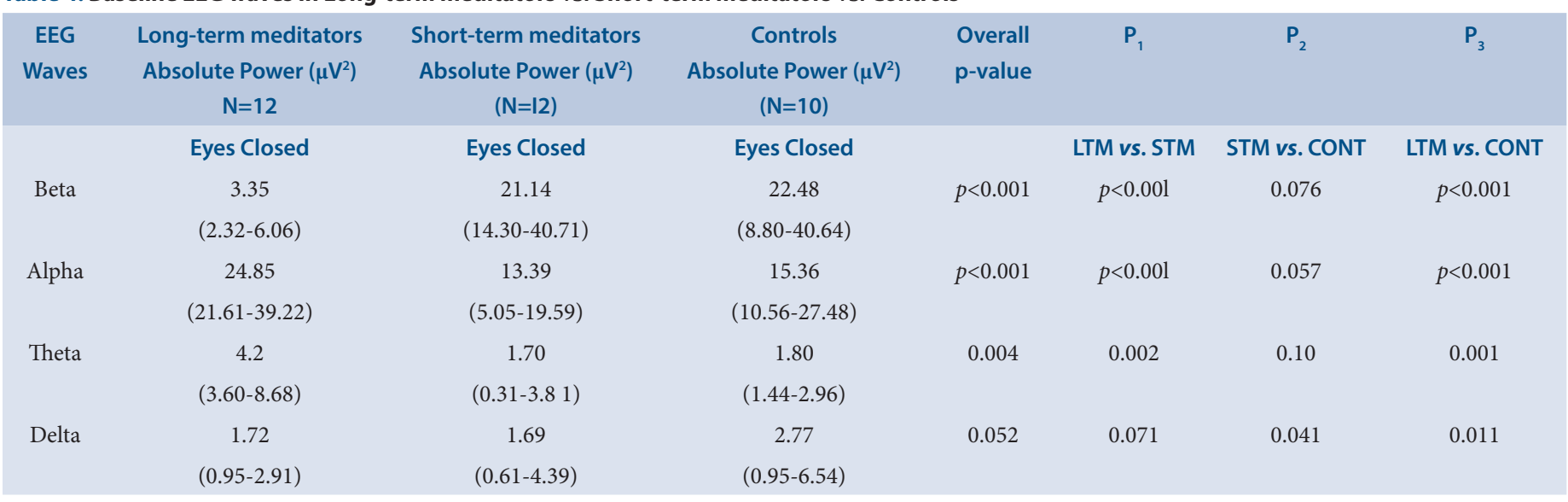

All the values are in median (range). One way ANOVA is used for the comparison among Long-term meditators $v$ s. Short-term meditators vs. Controls. $\mathrm{P}_{1}=$ Lon g-term meditators vs. Short-term meditators; $\mathrm{P}_{2}=$ Short-term meditators vs. Controls; $\mathrm{P}_{3}=$ Long-term meditators $v$ s. Controls 


\section{CONCLUSION}

Present findings might be useful in defining basic EEG features to differentiate meditators (long-term vs. short-term) based on the duration of practice of meditation and depth of meditation.

\section{ACKNOWLEDGEMENT}

Authors are thankful to Department of Biotechnology (DBT), Govt. of India, New Delhi to provide financial support for successfully completing the study. We would also like to acknowledge the IHC associates and staff for their contribution and support to run the regular programs at IHC, Department of Physiology, AIIMS, New Delhi, India.

\section{Financial support and sponsorship}

Department of Biotechnology (DBT), Govt. of India, New Delhi.

\section{CONFLICT OF INTEREST}

There are no conflicts of interest.

\section{ABBREVIATIONS}

LTMs: Long-term meditators; STMs: Short-term meditators; EEG: Electroencephalography.

\section{REFERENCES}

1. Dafoe T, Stermac L. Mindfulness Meditation as an Adjunct Approach to Treatment Within the Correctional System. J Offender Rehabil. 2013;52(3):198-216.

2. Hagerty MR, Isaacs J, Brasington L, et al. Case Study of Ecstatic Meditation: fMRI and EEG Evidence of Self-Stimulating a Reward System. Neural Plast. 2013;(2013):1-12

3. Faber $\mathrm{PL}$, Lehmann $\mathrm{D}$, Tei $\mathrm{S}$, et al. EEG source imaging during two Qigong meditations. Cogn Process. 2012;13(3):255-65.

4. Lagopoulos J, Xu J, Rasmussen I, et al. Increased theta and alpha EEG activity during nondirective meditation. J Altern Complement Med. 2009;15(11):1187-92.
5. Anand BK, Chhina GS, Singh B. Some aspects of electroencephalographic studies in yogis. Electroencephalogr Clin Neurophysiol. 1961;13(3):452-6.

6. Cahn BR, Delorme A, Polich J. Occipital gamma activation during Vipassana meditation. Cogn Process. 2010;11(1):39-56.

7. Ohana AB, Glicksohn J, Goldstein A. Mindfulness-induced changes in gamma band activity- Implications for the default mode network, self-reference and attention. Clin Neurophysiol. 2012;123(4):700-10.

8. Saggar M, King BG, Zanesco AP, et al. Intensive training induces longitudinal changes in meditation state-related EEG oscillatory activity. Front Hum Neurosci. 2012;6(256):1-14.

9. Hasenkamp W, Barsalou LW. Effects of meditation experience on functional connectivity of distributed brain networks. Front Hum Neurosci. 2012;6:38.

10. Paul J, Kannathal N, Sadasian K, et al. Complex electroencephalogram dynamics during meditation. JCCM. 2007;2(4):220-30.

11. Barnhofer T, ChittkaT, Nightingale $\mathrm{H}$, et al. State Effects of Two Forms of Meditation on Prefrontal EEG Asymmetry in Previously Depressed Individuals. Mindfulness. 2010;1(1):21-7.

12. Rael B, Cahn BR, Polich J. Meditation States and Traits: EEG, ERP and Neuroimaging Studies. Psychol Bull. 2006;132(2):180-211.

13. Yadav RK, Magan D, Mehta M, et al. Anxiety, Subjective Well-being and Personality is Improved Following a Comprehensive Short-term Yoga-based Lifestyle Intervention. Int J Yoga. 2012;5(2):134-9.

14. Aftanas LI, Golocheikine SA. Human anterior and frontal midline theta and lower alpha reflect emotionally positive state and internalized attention: Highresolution EEG investigation of meditation. Neurosci Lett. 2001;310(1):57-60.

15. Nair AK, Sasidharan A, John JP. Just a minute meditation: Rapid voluntary conscious state shifts in long term meditators. Conscious Cogn. 2017;53:176-84.

16. Banquet JP. Spectral analysis of EEG in meditation. Electroencephalogr Clin Neurophysiol. 1973;35(2):143-51.

17. Huang HY, Lo PC. EEG dynamics of experienced Zen meditation practitioners probed by complexity index and spectral measure. J Med Eng Technol. 2009;33(4):314-21.

Cite this article: Magan D, Yadav RK, Deepak KK. Comparative Analysis of Depth of Meditation Between Long-Term and Short Term Meditators using Electroencephalography. Int J Clin Exp Physiol. 2018;5(4):178-83. 